\title{
REFLEXIONES EN TORNO A LA COMPLEJIDAD COMO RUIDO METODOLÓGICO EN EL CAMPO DEL CONOCIMIENTO \\ Liliana Quintero*
}

RESUMEN: Este texto propone una reflexión en torno a la teoría de la complejidad como una manera distinta de comprender el conocimiento. Una de las críticas más relevantes es la problemática que encierra la escisión cartesiana de mente-cuerpo. Con ello se pretende mostrar cómo la complejidad nos permite introducir dispositivos que generan una vinculación con distintas disciplinas, lo que hace posible cuestionar problemas como la sustancialidad o la representación. La intención es integrar conceptos como enacción, no linealidad y medio ambiente a manera de vehículos para pensar de otra manera la relación entre la filosofía y la vida. Finalmente, se consideran casos específicos de científicos, artistas y humanistas que integran el pensamiento complejo como una forma vitalista y relacional para comprender la realidad.

PALABRAS CLAVE: complejidad, interdisciplina, información, enacción, sistema abierto, nueva epistemología.
ABSTRACT: In this article, we will propose the complexity theory as a different approach to understanding knowledge. One of its most relevant criticisms is the dilemma of the Cartesian split between mind and body. In this way, we attempt to show how complexity permits us to introduce tools producing a link between different fields thereby allowing us to question problems as sustainability and representation. Our intention is to integrate concepts as enaction, non-linearity, and the environment as tools to reflect differently on the relationship between philosophy and life. We will analyze specific instances of scientists, artists, and humanists utilizing this theory in a vital and relational way to understand reality.

KEYWORDS: complexity, interdisciplinary, information, enaction, open system, new epistemology.

* Taller de Investigación, Centro Multimedia, Centro Nacional de las Artes. 


\title{
REFLEXIONES EN TORNO A LA COMPLEJIDAD COMO RUIDO METODOLÓGICO EN EL CAMPO DEL CONOCIMIENTO
}

\begin{abstract}
La complejidad emerge como obscurecimiento, desorden, incertidumbre, antinomia. Esto mismo, que ha provocado la ruina de la fisica clásica, constituye la complejidad de la physis nueva. Lo que equivale a decir que [...] fecunda un nuevo tipo de comprensión y de explicación que es el pensamiento complejo [el cual] se forja $y$ se desarrolla en el movimiento mismo donde un nuevo saber sobre la organización y una nueva organización del saber se nutren mutuamente.
\end{abstract}

Edgar Morin

$E_{n}$ la actualidad es necesario abrir campos distintos en lo que se refiere a metodologías (si es que aún es pertinente nombrarlas de esta manera) para poder pensar y des-activar el fenómeno del conocimiento. No es sólo integrarlo a un campo interdisciplinario, sino incluir la misma estructura móvil de la vida al quehacer científico, humanístico y artístico-estético para que éstos discutan otros sistemas del saber.

Para tal fin, un dispositivo útil es el pensamiento filosófico; mas éste no puede permanecer aislado, debe vincularse a la práctica artística, al quehacer científico y al acontecer experiencial. El pensamiento posmo- derno brindó un camino relevante al anunciar, de manera crítica, el fundamento instrumental que dio origen el pensamiento moderno. El problema es que sólo quedó anunciado y con ello se continuó con la separación entre filosofia-humanidades, arte y ciencia.

Quizá una de las apuestas más interesantes sea la llamada nueva epistemología, que ha edificado, o intentado activar, el denominado pensamiento complejo. Edgar Morin, uno de los representantes más significativos de dicho pensamiento, plantea que una de las cuestiones más problemáticas es que el pensamiento filosófico y científico se han cimentado bajo sistemas cerrados, y ese para- 
digma invalida la comunicación con lo vivencial. Morin hace énfasis especialmente en que es necesario integrar a los sistemas vivientes, pero no como objetos, sino como máquinas autopoiéticas. ${ }^{1}$ Algunas ideas de la Modernidad sustentaron el pensamiento de la naturaleza como algo calculable. Los modernos equilibraron la idea de vida basada en lo inerte: lo vivo es lo mecánico; así, lo creado por el hombre y por las máquinas adquieren la misma magnitud y, más allá de eso, se genera un giro imprescindible: lo mecánico conquista la autoridad vital, mientras que la naturaleza es lo que carece de vida. Podemos observar la diferencia que marca Morris Berman en dos visiones sobre la naturaleza:

[En la Edad Media es] entendida por medio de lo concreto y lo cualitativo. La naturaleza es viva, orgánica, la observamos y hacemos deducciones de principios generales.

[En el siglo XVII es] entendida por medio de lo abstracto y lo cuantitativo. La naturaleza está muerta, es

${ }^{1}$ Las máquinas autopoiéticas son definidas como unidades por, y sólo por, su organización autopoiética: sus operaciones establecen sus propios límites en el proceso de autopoiesis. No ocurre así con las máquinas alopoiéticas, cuyos límites los fija el observador que, especificando las superficies de entrada y de salida, determina lo que es pertinente para su funcionamiento. Véase Humberto Maturana y Francisco Varela, De máquinas y seres vivos. Autopoiesis: la organización de lo vivo, 2005, Buenos Aires, Lumen, p. 69. mecánica y es conocida por medio de la manipulación (experimento) y de la abstracción matemática. ${ }^{2}$

Frente a esta ambivalencia de concepción de la naturaleza, la Modernidad refleja un estado emblemático que recae hasta nuestros días. Fritjof Capra afirma que el problema de la perspectiva mecanicista del universo es que "basó su visión de la naturaleza en la fundamental división entre dos reinos interdependientes y separados: el de la mente y el de la materia. El universo material, incluyendo los organismos vivos, era para René Descartes una máquina que podía ser enteramente comprendida analizándola en término de sus partes". ${ }^{3}$

De esta manera, era imposible plantear que los sistemas vivientes dependiesen de una relación constante a nivel material-energético $\mathrm{y}$, también, a nivel organizacionalinformacional. ${ }^{4}$ La separación entre mente y cuerpo inauguró una teoría del conocimiento que estaba basada en la separación total del mundo vivo. "El sujeto determina al objeto", fue la sentencia que anunció la revolución epistemológica de la Modernidad. El sujeto ilusorio representa

${ }^{2}$ Morris Berman, El cuerpo como espíritu, 1993, Santiago, Cuatro vientos, p. 130.

${ }^{3}$ Fritjof Capra, La trama de la vida. Una nueva perspectiva de los sistemas vivos, 1998, Barcelona, Anagrama, p. 85.

${ }^{4} \mathrm{Cfr}$. Edgar Morin, Introducción al pensamiento complejo, 2004, Barcelona, Gedisa, p. 78. 
la presencia dominadora de Occidente, que es el que determina la forma cuasi única de entender el mundo. Las leyes de la Razón son las que dictan el acontecer de los fenómenos para fijar en los objetos una imagen del mundo.

Heidegger planteó que el mundo se ha convertido en imagen, el mundo se desprende como espejo de la razón y se inscribe como representación. En este sentido, se puede afirmar que se produce al objeto y, así, el camino de la ciencia moderna se desarrolla generando no sólo una interpretación mecanicista del cosmos, sino una producción e imitación del mismo.

Martin Heidegger, en su texto "La época de la imagen del mundo", afirma que en la actualidad cuando hablamos de ciencia ésta tiene un sentido muy distinto al de doctrina, difiere de la scientia de la Edad Media, así como de la episteme de los griegos. La ciencia griega nunca fue exacta, porque su esencia así lo mostraba (en algún sentido, tampoco lo necesitaba). La ciencia moderna se construyó bajo los cimientos de la ciencia griega, Newton hubiera sido imposible sin Euclides, Arquímedes o Pitágoras. ${ }^{5}$ Esto es cierto, pero ¿cuál es la diferencia?, ¿qué es lo que encierra dicha ciencia y por qué aún nos preguntamos por ella?

${ }^{5} C f r$. Martin Heidegger, "La época de la imagen del mundo" en Caminos del bosque, 1996, Madrid, Alianza.
La diferencia entre el científico moderno y el científico griego es que éste conserva la congruencia entre los objetos naturales; aquél altera las cosas, construye modelos intelectuales que se oponen a los hechos tal como los presenta la experiencia inmediata. La ciencia moderna está fundada en la exactitud, es decir, el método de investigación está generado a partir de la física-matemática. La explicación de la naturaleza parte de los números y del cálculo. Pero, a partir de la herencia de los empiristas, la ciencia moderna se funda en la investigación y en el experimento, con base en leyes generales. La razón se hace legislativa de la experiencia. La ciencia moderna, de igual forma, está determinada por un tercer proceso fundamental: la empresa; en otras palabras, se institucionaliza. La idea de progreso está implícita en la ciencia moderna, por lo que tiene la obligación de dar resultados eficientes y exactos. ${ }^{6}$

A partir de Copérnico y Galileo surgen más que ideas brillantes, la razón humana sufre una transformación, lo que modifica la historia futura. La razón moderna adopta una nueva postura y se plantea nuevas metas que abarcan todo lo que es. El cambio alcanza todo resquicio de la existencia humana. Aun las ideologías políticas que dominan el mundo

${ }^{6}$ Cfr. ibid. 
moderno son diferentes a las que hubo en la antigüedad; aquéllas consideran al hombre amo activo de la naturaleza y, a partir de este dominio, se proponen transformar la totalidad de su existencia social. Actualmente, es evidente el vínculo esencial entre ciencia y tecnología. El pensamiento moderno problematizó la atribución de todo el poder al sujeto $\mathrm{y}$, de esta manera, la integración de la epistemología dentro de una esfera ontológica. Así, el sujeto queda establecido como un fantasma del universo objetivo al tratar "de revertir las perspectivas epistemológicas del sujeto, es decir, el objetivo del observador científico era, hasta el presente, eliminar la imprecisión, la ambigüedad, la contradicción". ${ }^{7}$

Uno de los filósofos más relevantes, cuyo pensamiento vislumbró está problemática, fue Hegel. Para él, la importancia no radica en la cosa en sí, sino en los momentos, tomando en cuenta el fluir dialéctico de cada uno de ellos. Asimismo, invierte la construcción filosófica al designar a la lógica como un sistema de razón pura, lo que la convierte en más que un instrumento del pensamiento, es su contenido la conciencia de la esencia para el espíritu. Hegel incluye la contradicción y la transformación como elementos fundamentales en su sistema filosófico; la primera es justamente la elevación de la razón sobre

\footnotetext{
${ }^{7}$ Morin, op. cit., p. 87.
}

las limitaciones del intelecto. Hegel parte de la multiplicidad; en este sentido, su pensamiento rompe con la estructura tradicional y abre un diálogo con la complejidad. Aunque vuelve a cerrar el sistema resolviéndolo desde la esfera del pensamiento, integra los paradigmas objetivista $\mathrm{y}$ subjetivista, dejándolos como momentos necesarios para otra transformación dialéctica.

Pero, ¿cómo salirnos de los límites del pensamiento?, ¿es posible pensar la realidad desde su sentido vivencial?, ¿cuáles son los límites de la epistemología cuando se piensa a sí misma? El problema es, quizá, que la filosofía aún sigue estructurándose desde el pensar mismo. La cuestión está en la manera en la que ejecutamos el ejercicio del pensamiento. ¿Cómo integrar la no-sustancialidad, la no-linealidad, la multiplicidad, la configuración activa de las redes? No sólo es pensar de otra manera, sino estar en el mundo de otra manera. Cuando Morin se refiere a que hay que poner atención en las relaciones heterogéneas, ya que este cruce de nodos permite una comprensión integral entre el pensar-actuar (siempre y cuando sea desde una perspectiva de sistemas abiertos), advierte dos consecuencias:

La primera es que las leyes de organización de lo viviente no son de 
equilibrio, sino de desequilibrio, retomando o compensando el dinamismo estabilizado [...] la segunda es que la inteligibilidad del sistema debe encontrarse no solamente en el sistema mismo, sino también con el ambiente, y esa relación no es simple dependencia, sino que es constitutiva del sistema. ${ }^{8}$

Posiblemente, la filosofía ya las había tomado en cuenta, pero fundamentó sólo desde parámetros teóricos. La intención es cuestionar cómo se integran otras maneras de pensar-actuar a los objetos del conocimiento. Edgar Morin abre la pregunta por la complejidad llevada al terreno del conocimiento y le interesa introducir un nuevo paradigma: la integración del ambiente como factor desestabilizador. La cuestión no es partir de la reflexión tradicional de la sustancialidad, sino poner en acción el flujo de relaciones y los acontecimientos desde su estado móvil. A simple vista, parece una obviedad que el eco-sistema funja como un factor de diálogo para la teoría del conocimiento, pero Morin intenta mostrar que el problema del objetivismo fue creer que las entidades del conocimiento y su comportamiento eran completamente estables, fijas y controlables. Sin embargo, si se formula una cuestión

\footnotetext{
${ }^{8}$ Ibid., p. 79.
}

dinámica en la cual el ambiente es más que un factor aislado de conocimiento, es decir, un factor que interactúa con nuestra evolución-involución química-biológica:

El eco-sistema natural, nos incita a examinar las características biológicas del conocimiento, esta Biología del conocimiento se ocupa evidentemente, de las formas cerebrales a priori constitutivas del conocimiento humano y también de sus modos de aprendizaje a través del diálogo con el ambiente [...] el punto de vista que nos sitúa en nuestro ecosistema social hic et nunc, el cual produce las determinantes/ condicionamientos ideológicos de nuestro conocimiento. ${ }^{9}$

Edgar Morin piensa que lo relevante será darle un sentido epistémico a la concepción abierta de la relación sujeto-objeto. No podemos omitir que el objeto se encuentra en un mundo abierto, el cual no se logra controlar en su totalidad y se escapa al determinismo estructural. ${ }^{10}$

Otro de los conceptos paradigmáticos en la actualidad es la información, matriz esencial de disciplinas como la teoría de sistemas y la cibernética. Uno de sus propósitos fue vincular fisiólogos y expertos en tecnología. Creó una nueva forma de entender la comunicación entre entorno, máqui-

\footnotetext{
${ }^{9}$ Ibid., p. 93.

${ }^{10}$ Ibid., p. 94.
} 
nas y ser humano, ya que abrió un campo esencial para la integración de los objetos de conocimiento y para su reacción con el mundo, no sólo como acción, sino como modelos de pensamiento, para poder inventar nuevas disciplinas. Gilbert Simondon define la información como:

[la] Extraña materia inmaterial en la que se basan todos los seres vivos artificiales, la información propulsada por la cibernética implica desembarazarse del tradicional esquema hilemórfico aplicado a la actividad técnica: hacer algo consiste en dar una forma a una materia inerte según una finalidad conocida por el hombre. La información muestra que las cuatro causas aristotélicas están condensadas en la materia misma y que dar forma, in-formar, le ocurre tanto a lo vivo como a lo artificial sin que la conciencia y la fuerza del hombre sean necesarias. ${ }^{11}$

Los sistemas cibernéticos pueden dirigir de manera automatizada a muchos otros conjuntos de artefactos. Hay una integración orgánica que es propia de los objetos naturales. La intención de la cibernética muestra un giro de la Modernidad:

\footnotetext{
${ }^{11}$ Rodríguez, MSOT, p. 20, apud María Ginette Múnera Barrios, "Pensamiento biopolítico en Gilbert Simondon. Técnicas y filosofía de la vida", en Iliana Hernández y Raúl Niño Bernal (eds.), Estética, vida artificial y biopolítica, 2010, Bogotá, Pontificia Universidad Javeriana, p. 214.
}

imitar lo vivo, lo orgánico, pero trasladado a las máquinas.

Morin forjó un vínculo entre el concepto de información y el de organización, y analizó la información como un modelo programático, en el cual el programa es un portador de información; ésta fue la aportación de Morin. Se puede considerar cierto proceso informacional que explora la posibilidad de calcular el dominio biológico. Desde que se estableció que la auto-representación de la célula o del organismo podía ser concebida a partir de una duplicación de un material genético, el ADN modifica nuestra manera de comprender la información, ya que se vuelve un concepto con una carga procesual. $\mathrm{Si}$ analizamos la configuración del ADN, en el que cada una de las letras tiene cierta información en sí misma y ésta, a su vez, es activada dependiendo de su combinatoria, se convierte en una unidad de sentido abierta y el desciframiento del ADN se traslada de "un terreno comunicacional a un terreno organizacional y así la información se considera organizacional ya sea como memoria, ya sea como mensaje ya sea como programa o más bien como todo a la vez". ${ }^{12}$

Con la integración de la efervescencia interdisciplinaria de los años 60 se puede observar cómo la ciber-

\footnotetext{
${ }^{12}$ Morin, op. cit., p. 81.
} 
nética dio origen a nuevos campos epistémicos y cómo se pudo aplicar de manera práctica el vínculo transdisciplinario. En este sentido, las máquinas complejas complementan algunas disciplinas, como la biología. Hoy en día, es imposible pensar los procesos computarizados y los procesos biológicos de manera separada, como afirma Jordi Vallverdú en su obra Bioética computacional: "La computación es algo intrínseco a la investigación científica contemporánea en muchas disciplinas, no un mero instrumento o ayuda". ${ }^{13}$

Lo que estamos presenciando es la integración de la cibernética a los procesos íntimos de lo científico, pero no para facilitarlos, sino como un híbrido. La información digital se vuelve crucial, se torna un elemento que posibilita los procesos de investigación. Se ha generado una dependencia entre los investigadores, las corporaciones, que son quienes financian, y el software, que aporta la ilusión-concreción de integrar imágenes digitales que permiten visualizar aquello que parecía imposible ver.

Otro concepto esencial para la modificación del paradigma tradicional es el de acción, que para Gilbert Simondon es uno de los eslabones para pensar y comprender el mundo

${ }^{13}$ Jordi Vallverdú, Bioética computacional, 2009, Barcelona, Fondo de Cultura Económica, p. 63. y su relación con el entorno naturalartificial, ya que el individuo no es un ser vivo, sino un acto, el individuo es ser agente de ese acto. El individuo es capaz de aumentar el contenido de la información. La individuación no es sustancia, sino modulación. Cada individuo reacciona de una manera autónoma; aunque herede información, se vincula de manera distinta.

Hay génesis de formas cuando la relación de un conjunto viviente con su medio y con él mismo pasa por una fase crítica rica en tensiones y en virtualidad, que concluye con la desaparición de la especie o con la aparición de una nueva forma de vida. El todo de la situación está constituido no solamente por la especie y su medio, sino también por la tensión del conjunto formado por la relación de la especie con su medio y en el cual las relaciones de incompatibilidad se vuelven cada vez más fuertes. De hecho, no es solamente la especie la que se modifica, sino que es también todo el conjunto del complejo vital formado por la especie y su medio el que descubre una nueva estructura. ${ }^{14}$

Finalmente, es importante mencionar otra interacción primordial que propone la complejidad en función del conocimiento: el azar; ese

${ }^{14}$ Simondon, La individuación psíquica $y$ colectiva, apud Múnera, op. cit., p. 217. 
aspecto de la incertidumbre que, como afirma Morin, coexiste dentro los linderos de nuestro entendimiento o ya desde los fenómenos. El azar exige un sinfín de caminos, de cruces no preestablecidos, pero también otra apertura a la causalidad predeterminada del conocimiento cerrado. Edgar Morin propone el concepto de ruido como vehículo que desestabiliza; cuestiona cómo la manera de conocimiento ha estado mediada por un sujeto que perturba, deforma la realidad. Ésta no es lo que aparece, pero tampoco es sólo lo que creemos conocer, hay una intermediación, por eso advierte que el "error" es creer que hay un conocimiento objetivo. ${ }^{15}$ En este sentido, la aportación de la complejidad en la biología permite que el trabajo interdisciplinario sea un factor primordial, no sólo en las aportaciones multidisciplinarias, sino en los cruces, en los espacios vacíos, en los cuales una disciplina deja de ser ella misma, como la física, la teoría de sistemas y las humanidades, que ponen constantemente aportaciones metodológicas, por ejemplo: "Las matemáticas en la actualidad discuten problemas que tienen como base la imprecisión y no sólo en el terreno de los fenómenos sino de los conceptos, los fuzzy sets, los conjuntos imprecisos". ${ }^{16}$

${ }^{15}$ Morin, op. cit., p. 89.

${ }^{16} \mathrm{Ibid}$, p. 87.

\section{Propuestas interdisciplinarias: usos de la complejidad como metodología emergente}

Se ha brindado un breve análisis de algunos de los conceptos que sirven de puente para pensar otras maneras de comprensión de lo real. La complejidad es, sin duda, una especie de dispositivo metodológico que nos permite problematizar las distintas aportaciones y cómo se pueden poner en acción ciertos conceptos provenientes de disciplinas que parecían destinadas a lo meramente instrumental. No obstante, también es importante cuestionar, desde la esfera de los procesos, cómo interviene la complejidad y cuál sería su relevancia.

El trabajo de investigación desarrollado por la bióloga mexicana Mariana Benítez es un ejemplo interesante, ya que integra el estudio interdisciplinario y propone un vínculo teórico-práctico de usos de herramientas configuradas desde los fundamentos conceptuales del pensamiento complejo. El estudio reciente de la biología evolutiva requiere de criterios distintos a los que se había enfrentado el estudio clásico. Al hacer énfasis en el desarrollo, se tienen que mirar otros campos, ya que el desarrollo no es proporcional y es altamente no lineal. Benítez propone:

La visión sistémica del desarrollo permite llevar a cabo análisis com- 
parativos en una versión dinámica y de sistemas, es decir, permite comparar no sólo las estructuras de un organismo sino los procesos a través de los cuales surgieron estas estructuras, lo que probablemente nos lleve a entender cómo surgió y cómo ha evolucionado el desarrollo mismo. ${ }^{17}$

Atender a los seres vivos como sistemas de interacciones dinámicas modifica el quehacer científico y presupone poner en acción factores móviles, como la aportación de la interacción del ambiente como elemento de estudio, pero enfatizando los aspectos ecológicos, sociales y económicos. Los estímulos ambientales generan una importancia en el desarrollo de la forma. El medio ambiente propone una plasticidad fenotípica, incluso la raíz de una planta depende de muchos factores que se regulan de manera no lineal: interacciones físicas, redes de regulación genética y estímulos ambientales.

Los sistemas complejos exhiben propiedades dinámicas y estructurales colectivas no definidas para las entidades individuales que los componen. Por ejemplo, las células que conforman el sistema nervioso del ser humano interactúan entre sí, y de las interacciones entre ellas (y otros factores) surge la mente. Sin embargo,

${ }^{17}$ Mariana Benítez, Desarrollo. La odisea del organismo, 2011, México, Centro de Ciencias de la Complejidad/UnAM, p. 53. no podemos decir que la mente pueda entenderse mediante el estudio aislado de una neurona, ni de la expresión genética en una de estas células; es necesario estudiar al sistema nervioso completo, a las neuronas y sus interacciones. Entonces, la hasta ahora eficaz y común estrategia de desmenuzar los sistemas de estudio tanto como se pueda, resulta insuficiente. El estudio de los sistemas complejos, y en particular, de los sistemas biológicos, concibe al organismo como un todo funcional y estructural en el que las partes interactúan afectándose unas a otras. ${ }^{18}$

La labor desarrollada por la doctora Benítez sorprende, ya que al cambiar de paradigma y llevarlo al estudio concreto, y al situar criterios como la disposición del ambiente como factor activo, la no linealidad, la modularidad, entre otros, supone una mirada crítica, pero que se activa desde la práctica científica.

Otra de las aportaciones significativas es la de los científicos Humberto Maturana, Francisco Varela, Evan Thompson y Eleanor Rosch, quienes en su estudio de las ciencias cognitivas han desempeñado un papel crítico y han reformulado su estructura metodológica al integrar la experiencia corporal. En su libro De cuerpo presente refieren que el conocimiento

${ }^{18}$ Ibid., p. 51. 
no se basa sólo en la exploración del pensamiento puro, sino que, al integrar al cuerpo como experiencia vivida, se convierte en un elemento esencial para la transformación del conocimiento. Su intención es dislocar la idea de sujeto y proponen añadir una "acción corporizada como sistema fenomenológico-biológico". ${ }^{19}$

$\mathrm{Su}$ interés es rescatar la idea de que el mundo no está separado de nosotros. La historia de la filosofía ha hallado, tanto en el paradigma objetivo como subjetivo, modelos de comprensión de la realidad, ya sea desde el observador incorpóreo o como una mente des-mundada, ${ }^{20}$ pero no logran integrar las posibilidades de comprensión del mundo en la actualidad.

La manera de conocer el mundo no parte de la representación de un mundo dado: ésta es una de las críticas de la movilidad desenfrenada de la época contemporánea. Pero ¿cómo pensar un mundo sin la representación? Una de las críticas interesantes para intentar salir de los estigmas

${ }^{19}$ Francisco Varela, Evan Thompson y Eleanor Rosch, De cuerpo presente. Las ciencias cognitivas y la experiencia humana, 2005, Barcelona, Gedisa, p. 17. El guión es mío, para indicar que no sólo es una relación, sino un agenciamiento entre lo biológico y lo fenomenológico: una hibridación.

${ }^{20}$ Término empleado por Varela que se refiere a la manera en que las ciencias cognitivas separaron la mente del entorno físico. de la representación son los modelos conexionistas, ${ }^{21}$ los cuales generalmente abordan el procesamiento de operaciones distribuidas, localizado y simbólico; es decir, operaciones que se extienden sobre una red de componentes y, así, derivan en la emergencia de propiedades globales resistentes a la disfunción local. Para los conexionistas, una representación consiste en la correspondencia entre un estado global emergente y las propiedades del mundo, no es una función de símbolos particulares. ${ }^{22}$ Sin embargo, para Fernando Varela el concepto primordial es el de enacción, ya que no es mirar el mundo desde la representación, sino desde el enfoque enactivo, que se refiere a la crítica filosófica de la idea de que la mente es un espejo de la naturaleza, e incluso aborda este problema desde el corazón de la ciencia. ${ }^{23}$ La enacción parte de hacer emerger la realidad.

Finalmente, es importante mencionar la labor del artista-matemático-programador Santiago Ortiz, ya que su trabajo transita desde la complejidad. Las obras de Santiago Ortiz tienen un vínculo muy interesante con la práctica programática, la estética visual-auditiva y la teoría. No

${ }^{21}$ Conexionismo es un concepto propuesto por J. A. Feldman y B. H. Ballard, en su texto "Connectionist Models and their Properties", Cognitive Science, núm 6, 1982, pp. 205-54.

${ }^{22}$ Varela et al., op. cit., p. 33.

${ }^{23}$ Ibid., p. 34. 
pretende ilustrar esta última y no son piezas que sólo visualizan y representan conceptos, sino que emergen aspectos de la teoría del caos. Cuestiones como el azar, la complejidad y la empatía son conceptos-puente en los cuales Ortiz hace presente su búsqueda estética. Su conocimiento programático le permite explorar el medio; no sólo lo usa como herramienta, sino que, como menciona Mariela Yeregui, genera zonas de concepto, en las cuales el artista se convierte en un facilitador de diversos contextos. Pero, quizá, más que zonas de concepto, se podría hablar de zonashíbridas, en las cuales se producen transferencias metodológicas, tanto por el cruce de disciplinas, como porque al entrar a campos transdisciplinarios se producen vacios que irrumpen como potencialidades de aproximación a lo real, abriendo un campo de transferencia interlineal en la que cada disciplina deja un hueco en la cual se pueden explorar nuevas posibilidades y se convierte así en algo distinto.

Varias de las obras de Santiago Ortiz nos muestran este quehacer. Al artista le interesan las relaciones y experimenta con ellas, usa los medios digitales para explorar estos espacios de encuentro, pero no como emulación. Aquí radicaría la diferencia. En los paradigmas tradicionales, aun en la robótica contemporánea, el interés principal es imitar los rasgos humanos. Por ejemplo, los autómatas del siglo XVIII tenían la finalidad de construir máquinas muy bellas que representaran una actividad humana, como escribir. El escritor ${ }^{24}$ era un muñeco cuyo interior escondía una maquinaría de reloj y cuya precisión no permitía error alguno, pero era muy distinto lo que se contemplaba y la función real de la maquinaria. Este ejemplo se puede trasladar hasta los robots digitales contemporáneos, como los desarrollados por el colectivo Robotlab, ${ }^{25}$ grupo de ingenieros y artistas alemanes que experimentan con robots industriales y les dan usos inesperados, como escribir La Biblia con tipografía del siglo XV.

Las obras de Santiago Ortiz no emulan, enaccionan; se agencian con la teoría, la programación y las relaciones reales que cohabitan el mundo de la web. Por ejemplo, en su obra Connections, ${ }^{26}$ ejemplifica la complejidad de las relaciones y va mostrando poco a poco sus vínculos de trabajo con su Twitter y, posteriormente, despliega un enjambre de

${ }^{24}$ Disponible en http://www.youtube.com/ watch? $=8$ ir12nHBLCM, consultado el 28 de noviembre de 2013.

${ }^{25}$ Disponible en http://vimeo.com/3834870, consultado el 28 de noviembre de 2013.

${ }^{26}$ Santiago Ortiz. Moebio, disponible en https:// www.vizify.com/santiagoortiz/connections?s=twitte $\mathrm{r} \& \mathrm{u}=110648 \& \mathrm{f}=3486 \& \mathrm{t}=$ connections_share_everyone, consultado el 5 de diciembre de 2013 . 
relaciones y la forma en que éstas se van contagiando, al grado que puede uno vincular su entorno de relaciones reales con la pieza.

Otro ejemplo es Mitozoos, un modelo interactivo de vida artificial creada con el objetivo de que los participantes, por medio de la experimentación y el juego, puedan entender la relación entre el código genético y la vida. El trabajo presenta una interfaz que permite a los participantes crear organismos virtuales, llamados "mitozoos"; esencialmente, codifica su ADN y luego presencia la evolución de esos organismos en un universo simulado. Estos mitozoos viven, se reproducen y mueren en un sistema que permite que su código genético continúe evolucionando, incluso puede mutar, generación tras generació n. En la pieza Mitozoos, la información genética se muestra como una serie de códigos de color, diseñada sobre la base química real del ADN. ${ }^{27}$

Santiago Ortiz intenta hacer una apuesta distinta y su mayor interés es vincular la complejidad del mundo con la complejidad codificada.

Toda obra de arte con código se enfrenta al humano. Aun cuando las

${ }^{27}$ Información extraída y traducida del sitio oficial de Santiago Ortiz, http://moebio.com, consultado el 28 de noviembre 2013. reacciones de la máquina sean monótonas y repetitivas, las del humano serán siempre diferentes en sentido fuerte de la palabra. Y si ya se ha señalado para la literatura y otras formas de arte, la obra no vive en ella misma sino en su lectura, la anomalía y el descontrol son simplemente inevitables. $^{28}$

Quizá lo más peligroso del pensamiento, las ciencias y el arte con estructuras deterministas e instrumentales es que se tornan ortopedia, difícil de fisurar. Por eso, la complejidad, como ruido metodológico, tiene muchos aspectos positivos que brindar en los terrenos del conocimiento. Principalmente, nos recuerda que ni el mundo es un objeto dado ni el sujeto es des-mundado, sino que ambos, sujeto-viviente y mundosistema-fenomenológico-biológico se encuentran en una co-creción, atendiendo al término complejidad: "complexus que significa lo que está tejido en conjunto". ${ }^{29}$ De esta forma, habrá que tejer en conjunto las ciencias, las humanidades, las artes y el acontecer de la experiencia vivencial, y así, reformular aquello que creímos conocer.

\footnotetext{
${ }^{28}$ Santiago Ortiz. "Descontrol y anomalia", en Liliana Quintero y Amanda Lemus, Fronteras nómadas, 2009, México, CONACUlta, p. 209.

${ }^{29}$ José Rozo Gauta, Teoría de sistemas y pensamiento complejo, 2010, Medellín, Fondo Editorial Biogénesis, p. 14.
} 
CITAM Derechos Reservados.

La reproducción total o parcial de este artículo se podrá hacer si el ITAM otorga la autorización previamente por escrito. 